Trauma Berufskrankh 2018 $20: 157-158$ https://doi.org/10.1007/s10039-018-0398-3

(c) Springer Medizin Verlag GmbH, ein Teil von Springer Nature 2018

CrossMark

\author{
A. Ekkernkamp ${ }^{1,2} \cdot$ D. Gümbel ${ }^{2} \cdot$ D. Stengel ${ }^{1,2}$ \\ 'BG Kliniken - Klinikverbund der gesetzlichen Unfallversicherung gGmbH, Berlin, Deutschland \\ ${ }^{2}$ Klinik für Unfallchirurgie und Orthopädie, BG Klinikum Unfallkrankenhaus Berlin gGmbH, Berlin, \\ Deutschland
}

\title{
Risiko und Sicherheit - warum wir das eine eingehen und das andere erhoffen
}

Die zweitwichtigste Stufe in der MaslowPyramide unmittelbar nach physiologischen Grundbedürfnissen ist Sicherheit $[1,2]$. Gemäß Sicherheitsreport 2018 des Centrums für Strategie und Höhere Führung und des Instituts für Demoskopie Allensbach 2018 (http://www. glh-online.com/Sicherheitsreport2018/) macht sich die „Bevölkerung aktuell insgesamt deutlich weniger Sorgen um ihre Sicherheit als noch 2016“.

Interessanterweise setzen wir uns in einer mobilen und globalisierten Welt immer häufiger potenziell lebensbedrohenden Risiken aus. Die Wahrscheinlichkeit für den Einzelnen, einen schwerwiegenden oder tödlichen Schaden im Straßen-, Schienen- und Luftverkehr zu erleiden, ist auf eine theoretische Größe geschrumpft. In der unbewussten Abwägung zum dominierenden Nutzen, z. B. innerhalb kürzester Zeit von A nach $B$ zu gelangen, akzeptieren wir dieses stochastische Restrisiko [3]. Dieses können wir individuell auch nicht mehr beeinflussen, sobald wir uns einmal auf die klaren Vorzüge der Exposition eingelassen haben.

Katastrophen sind selten, aber sie werden sich ereignen - sei es ein Flugzeugabsturz, ein Bahnunglück oder ein Massencrash auf einer Autobahn. Die solidarische Verantwortung besteht darin, neben der Etablierung einer interdisziplinären Sicherheitskultur und primärpräventiver Maßnahmen die Konsequenzen aus derartigen Ausnahmeereignissen für den Einzelnen und die Gesellschaft so gering wie möglich zu halten. Hierfür setzen sich Partner wie die Deutsche Gesetzli- che Unfallversicherung, der Konzern der BG Kliniken, die Deutsche Gesellschaft für Orthopädie und Unfallchirurgie, die Deutsche Gesellschaft für Unfallchirurgie, unzählige weitere (berufs)politische Organisationen und natürlich auch die Bundeswehr täglich ein.

Der Sicherheitsreport 2018 legte aber auch nahe, dass sich „36\% der Bundesbürger persönlich durch Terroranschläge bedroht fühlen“ und „in Zukunft mehrheitlich von einem zunehmenden Risiko hierdurch ausgehen“"

Terror ist ein in der Bundesrepublik Deutschland in der jüngeren Vergangenheit real erlebter Gefahrenkomplex, welcher keinen primärpräventiven Maßnahmen zugänglich ist. Wir müssen bei aller Nüchternheit im Umgang mit dem Thema sicherstellen, dass uns Terroranschläge selbst ungeahnten Ausmaßes nicht unvorbereitet treffen und wir diese professionell abfangen können. Eine demokratische Gesellschaft muss vorgeben, wie sie extremistischen Tätern oder Gruppen gegenübertritt.

Wir konnten renommierte Experten gewinnen, um die notwendige präund innerklinische Struktur- und Prozessqualität für einen Massenanfall von Verletzten (MANV) darzustellen und die spezifischen Anforderungen bei einem Terrorereignis (Terror-MANV) zu beleuchten. Ergänzt wird dies um Standards der frühzeitigen Gerinnungskontrolle und forensische Ableitungen aus dem Terroranschlag am Berliner Breitscheidplatz Weihnachten 2016.

Wir hoffen, dass Ihnen die Beiträge wertvolle Informationen für Ihre tägli- 


\section{Editorial}

che Praxis liefern und Sie motivieren, sich aktiv in die fachliche Diskussion einzubringen. Lassen Sie uns Ihre Meinung wissen.
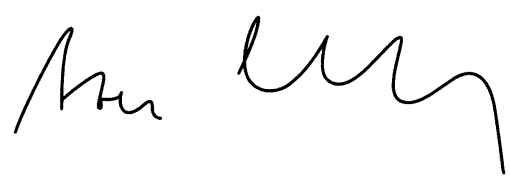

Axel Ekkernkamp

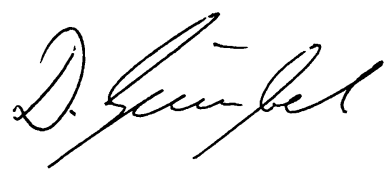

Denis Gümbel

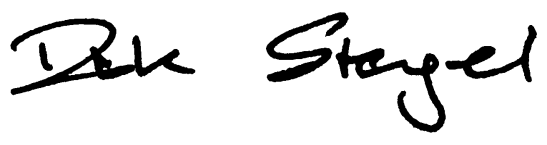

Dirk Stengel

\section{Korrespondenzadresse}

Prof. Dr. med. Dr. h.c.
A. Ekkernkamp
Klinik für Unfallchirurgie und
Orthopädie, BG Klinikum
Unfallkrankenhaus Berlin
gGmbH
Warener Str. 7, 12683 Berlin,
Deutschland

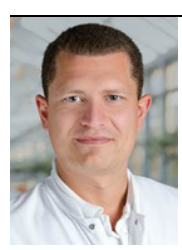

\section{Dr. D. Gümbel}

Klinik für Unfallchirurgie und Orthopädie, BG Klinikum Unfallkrankenhaus Berlin $\mathrm{gGmbH}$

Warener Str. 7, 12683 Berlin, Deutschland

Prof. Dr. D. Stengel, MSc
(Epi)
Klinik für Unfallchirurgie und
Orthopädie, BG Klinikum
Unfallkrankenhaus Berlin
gGmbH
Warener Str. 7, 12683 Berlin,
Deutschland
dirk.stengel@ukb.de

Interessenkonflikt. A. Ekkernkamp, D. Gümbel und D. Stengel geben an, dass kein Interessenkonflikt besteht.

\section{Literatur}

1. Maslow AH (1948) Higher and lower needs. JPsychol 25:433-436

2. Maslow AH (1948) Some theoretical consequences of basic need-gratification. J Pers 16:402-416

3. Wilde GJ (1998) Risk homeostasis theory: an overview. Inj Prev 4:89-91 